\title{
Editorial
}

\section{Robotic Artificial Intelligence in Dentistry}

Robotic Artificial Intelligence in Dentistry (RAID) will totally change the face of dentistry and the way clinical dental techniques are operated today. The advancement of digital equipment is use of robotic technology in dentistry. The key purpose of the clinical practice of robots is to sustain the meticulousness, superiority, and safety of surgical and nonsurgical procedures. The first surgical robot was introduced in 1992, but the technology had its first major breakthrough when the Da Vinci robot was approved by the US Food and Drug Administration (FDA) in 1997. In 1994, the First International Symposium on Medical Robotics and Computer Assisted Surgery was held in Pittsburgh, Pennsylvania, USA. The robot was programmed by a specialist from where all the robotic command was controlled and coordinated based on three-

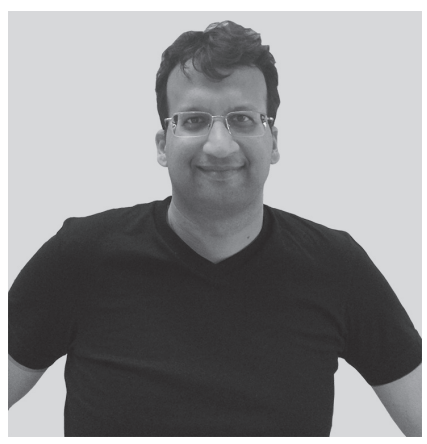
dimensional (3D) images of the operative area inside the patient produced by the audiovisual cameras. Robotic application will be applied and explored in all the clinical fields of dentistry including dental implants. The application will include the precise restoration of cavity, mapping the dentition, tooth preparation, crown cutting, bone cutting, oral stimulations, orthodontic tie-up, and esthetic cosmetic procedures including minimally invasive surgeries.

According to the World Health Organization (WHO) worldwide, 60 to $90 \%$ of schoolchildren and nearly $100 \%$ of adults have dental cavities. In operative dentistry, RAID would fundamentally control a dental drilling device united with a course-plotting method to determine the correct position of the tooth in relation to the patient. The robotic arm would either be operated directly by a dentist or be preprogrammed to accomplish its tasks based on imaging data/3D scan. The WHO also reported that globally about 30\% of people aged 65 to 74 years have no natural teeth. In the field of prosthodontics, RAID would be used to make digital impressions through an intraoral scanner/bioprinter. This data would then be transferred to the lab to produce temporary crowns or bridges in a very short time using a milling machine and to manufacture the final restorations in much shorter time than with conventional procedures. A robotic guidance system (YOMI) for dental implant procedures received FDA clearance that is a computerized navigational system intended to aid in both the planning (preoperative) and surgical (intraoperative) phases of dental implantation surgery. The system reportedly provides software to preoperatively plan dental implantation procedures and is designed to provide navigational guidance of the surgical instruments.

Future of RAID is extremely promising and new innovation and technologies are changing the dimension of dentistry everyday; RAID will definitively elevate the accuracy, predictability, safety, and quality of dental treatment in the next few years.

Rajiv Saini Editor-in-Chief International Journal of Experimental Dental Science

Associate Professor

Department of Periodontology \& Oral Implantology Pravara Institute of Medical Sciences Loni, Maharashtra, India 